\title{
Improved OAM-Based Radar Targets Detection Using Uniform Concentric Circular Arrays
}

\author{
Mingtuan Lin, ${ }^{1,2}$ Yue Gao, ${ }^{2}$ Peiguo Liu, ${ }^{1}$ and Jibin Liu ${ }^{1}$ \\ ${ }^{1}$ College of Electronic Science and Engineering, National University of Defense Technology, Changsha 410073, China \\ ${ }^{2}$ School of Electronic Engineering and Computer Science, Queen Mary University of London, London E1 4NS, UK \\ Correspondence should be addressed to Mingtuan Lin; linmingtuan08@163.com
}

Received 17 January 2016; Revised 8 April 2016; Accepted 4 May 2016

Academic Editor: Diego Masotti

Copyright (c) 2016 Mingtuan Lin et al. This is an open access article distributed under the Creative Commons Attribution License, which permits unrestricted use, distribution, and reproduction in any medium, provided the original work is properly cited.

\begin{abstract}
Without any relative moves or beam scanning, the novel Orbital-Angular-Momentum- (OAM-) based radar targets detection technique using uniform concentric circular arrays (UCCAs) shows the azimuthal estimation ability, which provides new perspective for radar system design. However, the main estimation method, that is, Fast Fourier Transform (FFT), under this scheme suffers from low resolution. As a solution, this paper rebuilds the OAM-based radar targets detection model and introduces the multiple signal classification (MUSIC) algorithm to improve the resolution for detecting targets within the main lobes. The spatial smoothing technique is proposed to tackle the coherent problem brought by the proposed model. Analytical study and simulation demonstrate the superresolution estimation capacity the MUSIC algorithm can achieve for detecting targets within the main lobes. The performance of the MUSIC algorithm to detect targets not illuminated by the main lobes is further evaluated. Despite the fact that MUSIC algorithm loses the resolution advantage under this case, its estimation is more robust than that of the FFT method. Overall, the proposed MUSIC algorithm for the OAM-based radar system demonstrates the superresolution ability for detecting targets within the main lobes and good robustness for targets out of the main lobes.
\end{abstract}

\section{Introduction}

Orbital Angular Momentum (OAM) has been widely studied in the optic regime regarding imaging, microscopic particle, and communication $[1,2]$. However, OAM in radio frequency band had not been developed until 2007 when the feasibility of OAM generating in a low-frequency band was proved in [3]. Unlike the common planar wavefront in the far field, the twisted beam carrying OAM has a helical shape of wavefront known as a vortex. Benefiting from its peculiar properties such as the orthogonality of integer topological modes and larger degrees of freedom, OAM-based communication and radar techniques show a promising potential.

Previous works about radio OAM mainly focused on communication systems consisting of two subfields, namely, performance analysis and design of antennas to generate twisted beams [4-9], and OAM multiplexing [10-13]. Radar based on this innovative concept had not attracted much attention until Guo et al. proposed OAM-based target detection model using the uniform circular array (UCA) in [14]. As depicted in Figure 1, instead of producing planar wave in a particular direction, the OAM-based radar employed twisted beams carrying different OAM modes to illuminate a target. This scenario provided a new perspective to estimate azimuthal information of targets without any relative moves or beam scanning as the traditional way required. Later, the detection model was further extended with Multiple Input Multiple Output (MIMO) and Multiple Input Single Output (MISO) schemes in [15]. Due to intrinsic drawbacks of the model, both schemes suffered from ambiguous problems, which require prior information involved as a solution. Under these two schemes, targets were usually not illuminated by main lobes of twisted beams, leading to a significant energy loss of echo signals. To overcome this problem, several uniform concentric circular arrays (UCCAs) were deployed to enhance the energy of received signals in [16], with each 


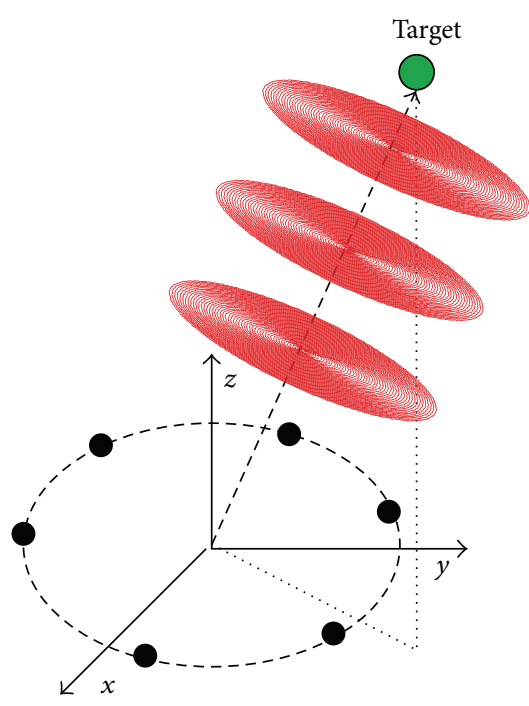

(a)

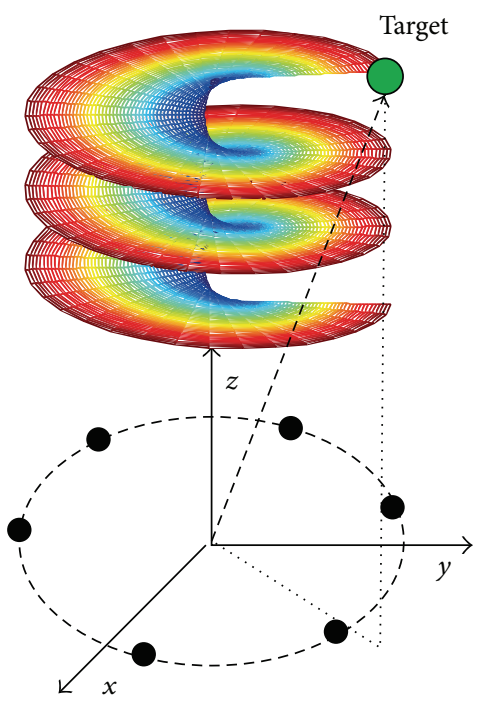

(b)

FIGURE 1: Target illuminated by (a) the traditional beam with planar wave and (b) the twisted OAM beam with helical wavefront.

ring producing a particular OAM mode and ensuring that all main lobes were pointed at the same direction. This scenario benefited the detection for targets within main lobes in the noisy environment. Nevertheless, the low-resolution estimation method FFT were not able to identify targets within main lobes but with a narrow azimuth gap. Additionally, the performance of detection for targets not illuminated by the main lobes was not analyzed.

Based on the scenario in [16], this paper reconstructs the model for detecting targets within the main lobes for superresolution by using the MUSIC algorithm [17]. Furthermore, the estimation performance of targets being out of main lobes is further analyzed.

\section{Current OAM-Based Detection Models}

The MIMO and MISO schemes of OAM-based radar techniques were investigated in [15]. For the MIMO scheme, the output summates the echo signals of all elements with the designed weights and a normalized real-time echo signal output of $M$ targets can be extended and expressed as follows:

$$
\begin{aligned}
& E(\alpha, t) \\
& =\sum_{m=1}^{M} \sigma_{m} \frac{e^{j 2 k r_{m}}}{r_{m}^{2}} e^{j 2 \alpha \varphi_{m}} J_{\alpha}^{2}\left(k a \sin \theta_{m}\right) s(t)+n(\alpha, t),
\end{aligned}
$$

where $\alpha$ is the OAM mode, $k$ is the wave number, $J_{\alpha}$ is the $\alpha$ th Bessel function, $a$ is the radius of circular array, $s(t)$ is the transmitted signal, $n(\alpha, t)$ is the real-time noise for OAM mode $\alpha$, and $r_{m}, \theta_{m}, \varphi_{m}$, and $\sigma_{m}$ refer to the distance, elevation, azimuth angle, and the radar cross section (RCS) of $m$ th target. For the MISO scheme, multiple antennas are employed at the transmitter to generate the twisted beams, while only one element is installed in the original point to

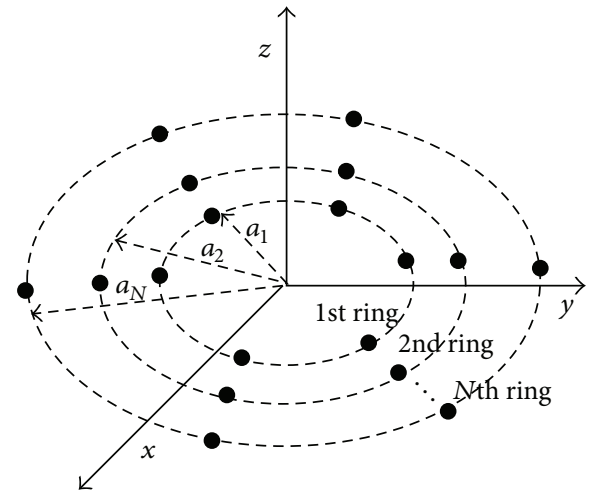

FIGURE 2: Uniform concentric circular arrays.

receive the echo signals. The normalized echo signals are given by

$$
\begin{aligned}
& E(\alpha, t) \\
& \quad=\sum_{m=1}^{M} \sigma_{m} \frac{e^{j 2 k r_{m}}}{r_{m}^{2}} e^{i \alpha \varphi_{m}} J_{\alpha}\left(k a \sin \theta_{m}\right) s(t)+n(\alpha, t) .
\end{aligned}
$$

By observing (1) and (2), a similar Fourier Transform relation lies in $\alpha$ and $\varphi$ regimes, which leads to the derivation of the FFT [14-16] to make an estimation of the azimuth angles of targets. Both MISO and MIMO schemes can not ensure that targets are illuminated by main lobes of different OAM modes, which make it vulnerable to the background noise.

$N$ UCCAs with formulated apertures $a_{i}, i \in[1, N]$, as shown in Figure 2 were proposed to engender $N$ OAM modes in [16] to tackle the above problem. In this scenario, larger aperture arrays in outer rings are used to generate higher modes twisted beams while inner rings create lower modes 
twisted beams, to make sure that targets can be illuminated by main lobes of each mode.

The normalized received echo signal using the UCCAs under the MIMO scheme can be written as follows:

$$
\begin{aligned}
E(\alpha, t)= & \sum_{m=1}^{M} \frac{\sigma_{m}}{r_{m}^{2}} J_{\alpha}^{2}\left[k a(\alpha) \sin \theta_{m}\right] e^{j 2 \alpha \varphi_{m}} e^{j 2 k r_{m}} s(t) \\
& +n(\alpha, t),
\end{aligned}
$$

where $a(\alpha)=(1.02 \alpha+1.874) / k \sin \theta_{0}$ refers to radius of the UCCA to create $\alpha$ mode with main lobe pointing at elevation angle $\theta_{0}$. Similar conclusions can be obtained for the MISO scheme.

\section{Proposed Estimation Based on the Music Algorithm}

Despite the fact that detection technique using the UCCAs could enhance the echo signal for targets within main lobes, the low-resolution estimation method FFT limits its application for detecting targets with narrow azimuth angle gaps. For targets within main lobes, this section rebuilds the model to achieve the superresolution by using the MUSIC algorithm. It is assumed that the prior information of target number $M$ is known.

3.1. MIMO Scheme. According to (3), despite the fact that main lobes of all OAM modes are pointing at $\theta_{0}$, there still exists echo energy difference for different modes. The energy differences can be eliminated by adjusting the array feeding gain $b(\alpha)$ for each mode; then the received echo signal is

$$
\begin{aligned}
& E(\alpha, t) \\
& \qquad \sum_{m=1}^{M} \frac{\sigma_{m}}{r_{m}^{2}} b(\alpha) J_{\alpha}^{2}\left[k a(\alpha) \sin \theta_{m}\right] e^{j 2 \alpha \varphi_{m}} e^{j 2 k r_{m}} s(t) \\
& \quad+n(\alpha, t) .
\end{aligned}
$$

Since targets are within main lobes, $\theta_{m}=\theta_{0}$. By designing the feeding gain of arrays for each mode

$$
b(\alpha)=\frac{c}{J_{\alpha}^{2}\left[k a(\alpha) \sin \theta_{0}\right]}
$$

thus (4) can be reformed as follows:

$$
E(\alpha, t)=\sum_{m=1}^{M} e^{j 2 \alpha \varphi_{m}} c \frac{\sigma_{m}}{r_{m}^{2}} e^{j 2 k r_{m}} s(t)+n(\alpha, t),
$$

where $c$ is a constant. $E(\alpha, t)$ involves the data in terms of the $\alpha$ regime and $t$ regime. By observing $e^{j 2 \alpha \varphi_{m}}, \varphi_{m}$ in $\alpha$ regime can be understood as the angular frequency in time domain. Therefore, the estimation of $\varphi_{m}$ in $\alpha$ domain is similar to estimate the angular frequency in time domain. Assume $f_{\alpha}$ is the sample rate in $\alpha$ regime; then according to the Nyquist sampling theory, to estimate $\varphi_{m}$ without aliasing,

$$
f_{\alpha} \geq 2 f_{\max }=2 \frac{w_{\max }}{2 \pi}, \quad w_{\max } \in[-2 \pi, 2 \pi],
$$

where $f_{\max }$ and $w_{\max }$ indicate the max frequency and angular frequency in $\alpha$ regime; hence $f_{\alpha} \geq 2$. The echo signals under different OAM modes are not sampled simultaneously, which is not applicable for the MUSIC algorithm. Fortunately, the time slot of the adjoined OAM modes is known, which could be calibrated to achieve the equivalent simultaneous sampling performance by delaying the corresponding time slot of $s(t)$ for specific OAM mode. Consider the calibrated real-time echo signals under $N$ OAM modes; then the echo signal vector can be gained as follows:

$$
\begin{aligned}
& \mathbf{E}=\left[\begin{array}{c}
E\left(\alpha_{1}, t\right) \\
\vdots \\
E\left(\alpha_{N}, t\right)
\end{array}\right]=\mathbf{A S}+\mathbf{n} \\
& =\left[\begin{array}{ccc}
e^{j \alpha_{1} 2 \varphi_{1}} & \cdots & e^{j \alpha_{1} 2 \varphi_{M}} \\
\vdots & & \vdots \\
e^{j \alpha_{N} 2 \varphi_{1}} & \cdots & e^{j \alpha_{N} 2 \varphi_{M}}
\end{array}\right]\left[\begin{array}{c}
\frac{c \sigma_{2} e^{j 2 k r_{1}} s(t)}{r_{1}^{2}} \\
\vdots \\
\frac{c \sigma_{M} e^{j 2 k r_{M}} s(t)}{r_{M}^{2}}
\end{array}\right] \\
& +\left[\begin{array}{c}
n\left(\alpha_{1}, t\right) \\
\vdots \\
n\left(\alpha_{N}, t\right)
\end{array}\right]
\end{aligned}
$$

where $\mathbf{A} \in C^{N \times M}$ is the steering vector in $\alpha$ regime, $\mathbf{S}=$ $\left[c \sigma_{1}\left(e^{j 2 k r_{1}} / r_{1}^{2}\right) s(t), \ldots, c \sigma_{M}\left(e^{j 2 k r_{M}} / r_{M}^{2}\right) s(t)\right]^{T} \in C^{M \times L}$ refer to echo signals of targets, and $L$ is the real-time sample length of echo signals in the time domain. From (8), it can be observed that the steering vector $\mathbf{A}$ is only related to the OAM modes $\alpha$ and $\varphi$, independent of the array shapes. For the direction of arrival (DOA) estimation, the steering vector depends on the space sampling method of the antenna, that is, the array shapes, while for the proposed model using the UCCAs to estimate the azimuth angles the steering vector is determined by the sampling method in $\alpha$ regime such as the uniform or sparse sampling. Under this proposed model, one OAM mode resembles one antenna in traditional DOA estimation model.

For presenting Gaussian noise, the covariance matrix of the echo signals under different OAM modes can be acquired by

$$
\mathbf{R}_{\mathbf{E}}=\mathbf{A R}_{S} \mathbf{A}^{H}+\rho_{n} \mathbf{I},
$$

where $\rho_{n}$ is the noise power. Similar to DOA estimation, columns of $\mathbf{A}$ are independent; however the echo signals of multiple targets are fully coherent, failing to meet the uncorrelated condition for the MUSIC algorithm. Obviously, $\operatorname{rank}\left(\mathbf{R}_{S}\right)=1$, which leads the leak from the signal subspace to the noise subspace and contaminates the orthogonality, deteriorating the performance of MUSIC. For traditional DOA estimation, spatial smoothing technique is utilized to tackle the coherent problem, which is only applicable for a uniform linear array (ULA), that is, uniform space sampling 




FIGURE 3: Front-spatial smoothing in $\alpha$ regime.

array. Regarding the proposed OAM-based radar model, the uniform linear OAM mode sampling in $\alpha$ regime is similar to the case of ULA for conventional DOA estimation. Therefore, the spatial smoothing is applicable to solve the coherent problem of the proposed model with uniform sampling. With appropriate reforms, other methods used in DOA estimation such as matrix decomposition can also solve the coherent problem in the proposed model. The spatial front smoothing technique is chosen in this paper to conquer the coherent problem.

According to the smoothing theory [18], divide N OAM sample modes in $\alpha$ domain to $p$ mixed subsamples blocks as shown in Figure 3. Each block has $h$ modes, so $N=p+h-$ 1. Subsequently, calculate the modified covariance matrix as follows:

$$
\mathbf{R}^{f}=\frac{1}{p} \sum_{i=1}^{p} \mathbf{R}_{i} \quad \mathbf{R}^{f} \in C^{h \times h}
$$

where $\mathbf{R}_{i}$ is the covariance matrix of $i$ th subblock $\mathbf{E}_{i}$. To achieve the full rank of signal space, the number of blocks should meet $p \geq M$.

The whole procedure of the spatial smoothing MUSIC algorithm to estimate the azimuth angles is listed as follows:

(1) Obtain $p$ original covariance matrix $\mathbf{R}_{i}(i=1,2$, $\ldots, p)$.

(2) Gain $\mathbf{R}^{f}$ based on (10).

(3) Make engine value decomposition of $\mathbf{R}^{f}$.

(4) Calculate the azimuth spectrum

$$
P(\varphi)=\frac{1}{\mathbf{a}^{H}(\varphi) \mathbf{V}_{n} \mathbf{V}_{n}{ }^{H} \mathbf{a}(\varphi)},
$$

where $\mathbf{V}_{n}$ is the noise subspace vectors obtained from the engine value decomposition of $\mathbf{R}^{f}$ and $\mathbf{a}(\varphi)=$ $\left[e^{j 2 \alpha_{1} \varphi}, \ldots, e^{j 2 \alpha_{N} \varphi}\right]^{T}$ is the search steering vector.

3.2. MISO Scheme. Similar conclusions can be retained for MISO scheme, but with several differences as follows:

(1) To avoid aliasing, the minimum sample rate of MISO scheme is half that of MIMO scheme.
(2) The resolution ability of MIMO scheme is double that of MISO scheme, with $\delta \varphi \propto 1 / 2 \alpha$ for MIMO scheme and $\delta \varphi \propto 1 / \alpha$ for MISO scheme.

(3) The searching steering vector is $\mathbf{a}(\varphi)=\left[e^{j \alpha_{1} \varphi}\right.$, $\left.\ldots, e^{j \alpha_{N} \varphi}\right]^{T}$.

\section{Analysis for Targets out of Main Lobes}

The targets detection within the designed main lobes is described in Section 3; however for those objectives out of main lobes, $b(\alpha) J_{\alpha}^{2}[k a(\alpha) \sin \theta]$ or $b(\alpha) J_{\alpha}[k a(\alpha) \sin \theta]$ is not a constant for all modes. Would the proposed MUSIC algorithm estimate azimuthal information accurately? There is no doubt that for a windowing real-time signal with different frequencies MUSIC can calculate the spectrum successfully. Similarly, $b(\alpha) J_{\alpha}^{2}[k a(\alpha) \sin \theta]$ and $b(\alpha) J_{\alpha}[k a(\alpha) \sin \theta]$ can be regarded as a window for $e^{j 2 \alpha \varphi}$ and $e^{j \alpha \varphi}$ specifically. Consequently the MUSIC algorithm can gain a satisfactory estimation as predicted. But the influence caused by the window should be analyzed. Since the influences for MIMO and MISO schemes are similar, to avoid the repeated discussion, the following analyses are made under the MIMO scheme.

According to (3) and (4), the influence of estimation brought by the square Bessel function or its reformation can be seen as the influence of the point spread function (PSF) of the windowing. Here we consider the azimuth profile of PSF as

$$
F(\varphi)=\int b(\alpha) J_{\alpha}^{2}(k a(\alpha) \sin \theta) e^{-j \alpha \varphi} d \alpha .
$$

According to signal processing theory, the multiplication relation in $\alpha$ regime indicates the convolution in $\varphi$ field. By analyzing the profile of PSFs, the estimation performance of targets out of main lobes can be evaluated. Figure 4 compares patterns of PSFs for targets with $\theta=14^{\circ}$ and $\theta=34^{\circ}$ specifically, using the same UCAA configuration with main lobes pointing at $\theta_{0}=14^{\circ}$. It can be observed for target not illuminated by main lobes that there are two high side lobes as shown in Figure 4(b), while no side lobes in Figure 4(a) appear for the target within main lobes. Those side lobes will introduce pseudo estimations into the azimuth spectrum, leading to ambiguity problems for target detection. Influences caused by different array configurations to estimation can be also observed by figures of different PSFs as shown in Figure 5. Comparing Figures 4 and 5, it can be implied that azimuthal estimation based on the UCCAs for targets out of main lobes will suffer the same ambiguity problem as a UCA configuration presented in [15].

\section{Simulation}

Simulation is presented to compare performances of the existing FFT method and the proposed MUSIC algorithm. Figure 6 illustrates comparisons of estimation performance between MIMO and MISO scheme and between MUSIC and FFT method for targets within the main lobes. It can be observed that MUSIC has distinct higher resolution ability than that of FFT method for both schemes while MIMO 


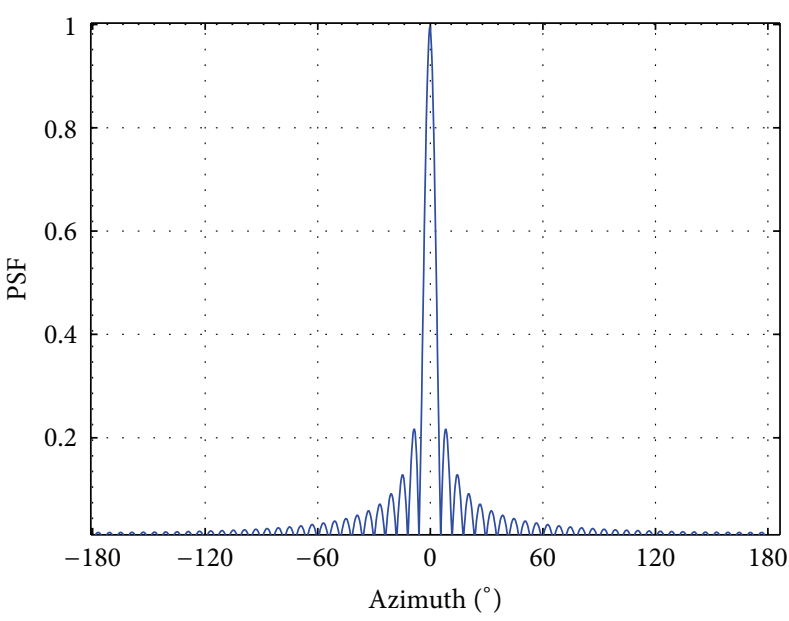

(a)

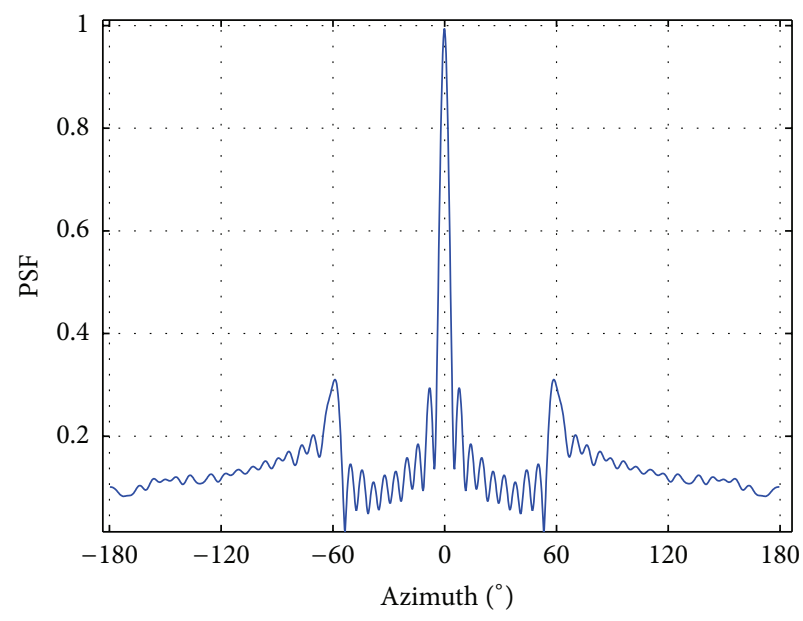

(b)

FIGURE 4: Azimuth profile of PSF using the same UCCA configuration with main lobes aimed at $14^{\circ}$ to illuminate targets with elevation: (a) $\theta=14^{\circ}$ and (b) $\theta=34^{\circ}$.

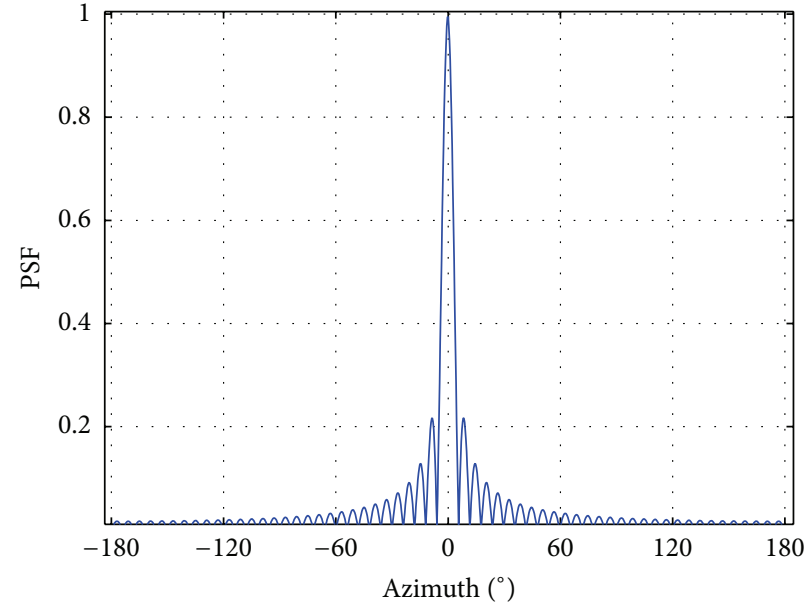

(a)

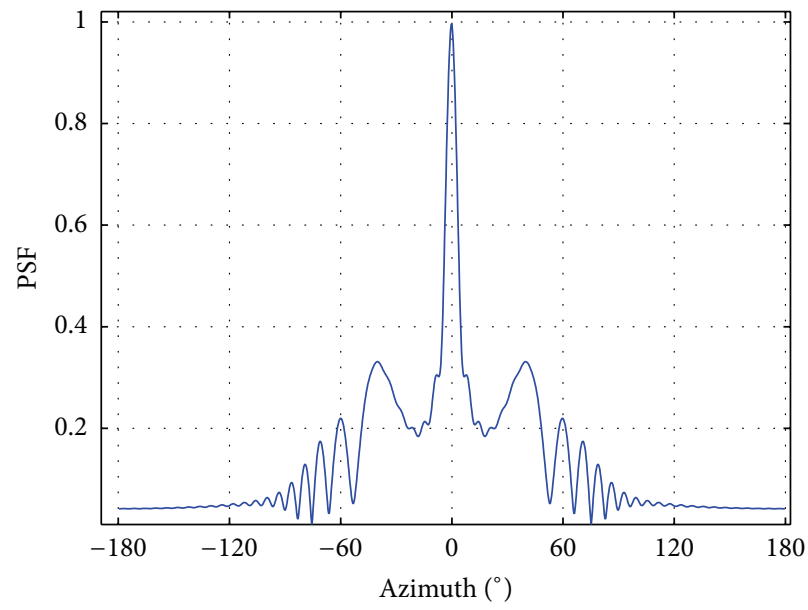

(b)

FIGURE 5: Azimuth profile of PSF of the same target with $\theta=14^{\circ}$ using (a) UCCA with $\theta_{0}=14^{\circ}$ and (b) UCA with a radius equal to the outer ring of UCCA.

scheme has a resolution advantage over that of MISO scheme. Specifically, the curve in Figure 7 gives the detailed information about the resolution of two schemes with two methods. Almost double resolution ability of MIMO scheme compared to MISO scheme can be viewed in Figure 7. By comparing Figures 7(a) and 7(b), the superresolution capacity of MUSIC for detection of targets within main lobes was apparently observed.

Using UCCA configuration as Figure 4 to illuminate two targets out of main lobes, four large ambiguous peaks appear in the spectrum of FFT in Figure 8(a), in accordance with the side lobes of PSF in Figure 4. Side lobes in Figure 8(b) also arise in MUSIC method but with great suppressions, which can be almost ignored. By comparing Figures 8(a) and 8(b), it can be found that the MUSIC algorithm has no great resolution advantage over FFT but instead is more robust than FFT with less ambiguity influence.

\section{Conclusion}

For the Orbital-Angular-Momentum- (OAM-) based radar system using uniform concentric circular arrays (UCCAs), this paper addressed the multiple signal classification (MUSIC) algorithm to improve the target detection performance. In comparison with the traditional FFT method, the proposed MUSIC algorithm achieved the superresolution for detecting the targets within the main lobes and good 


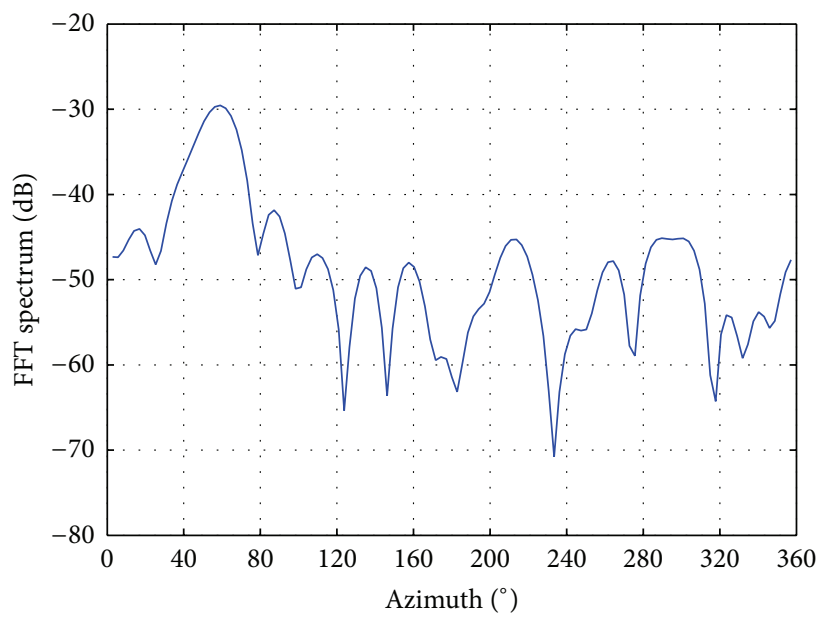

(a)

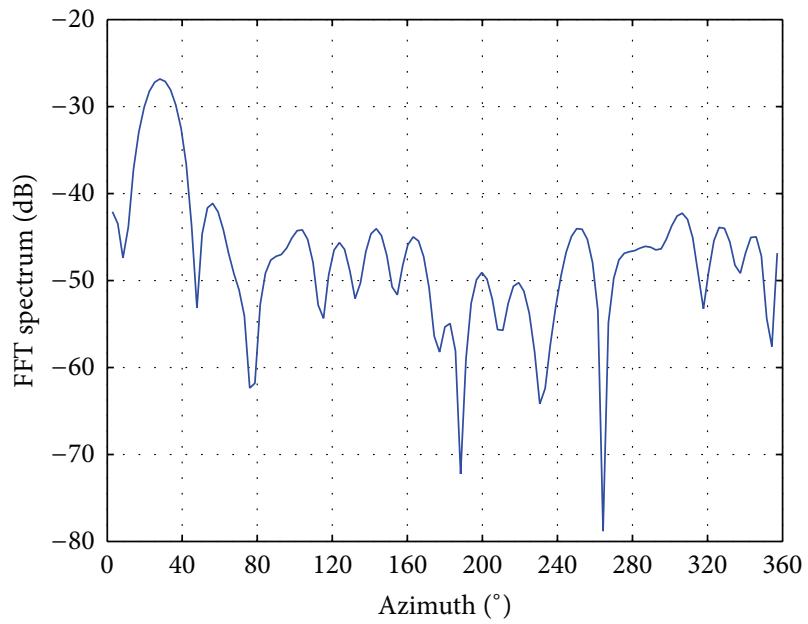

(c)

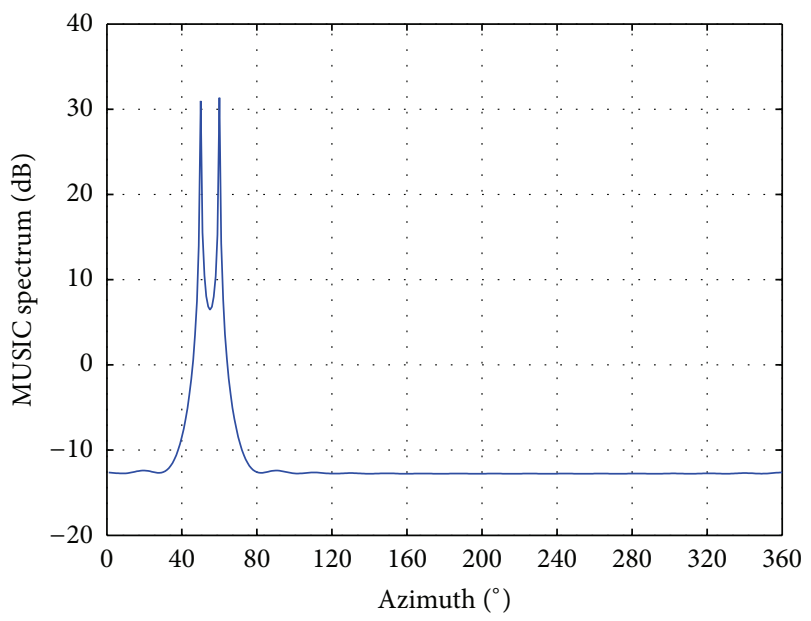

(b)

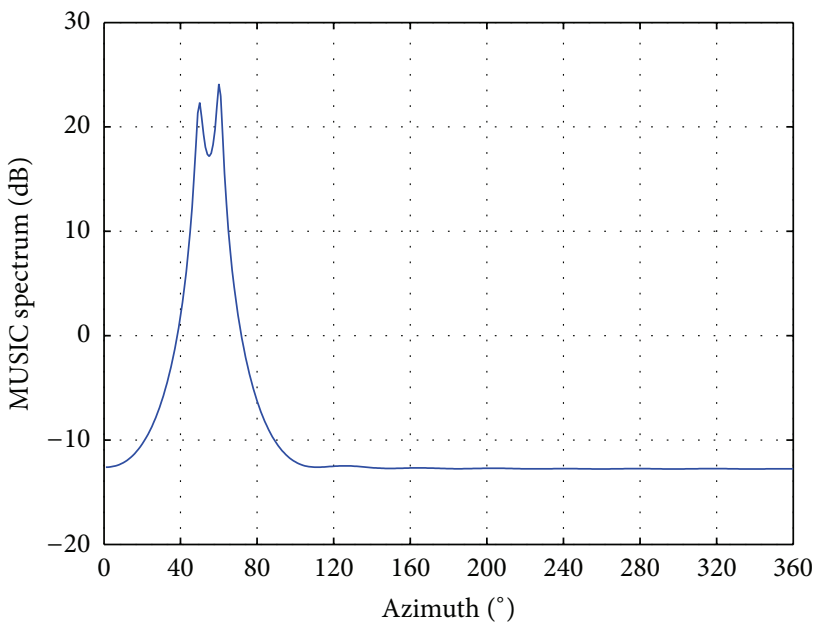

(d)

FIGURE 6: Azimuth angle estimation spectrum under MIMO scheme: (a) and (b); MISO scheme: (c) and (d). (a) and (c) use the FFT method, while (b) and (d) utilize the MUSIC algorithm. Two targets at $782.4 \lambda$ and $689.3 \lambda$ with the same elevation angle $14^{\circ}$ but different azimuth angles $50^{\circ}$ and $60^{\circ}$ are under illumination of main lobes of the UCCAs with configuration $N=60, f_{\alpha}=2$, and $L=1024$ at SNR $=10 \mathrm{~dB}$ environment.

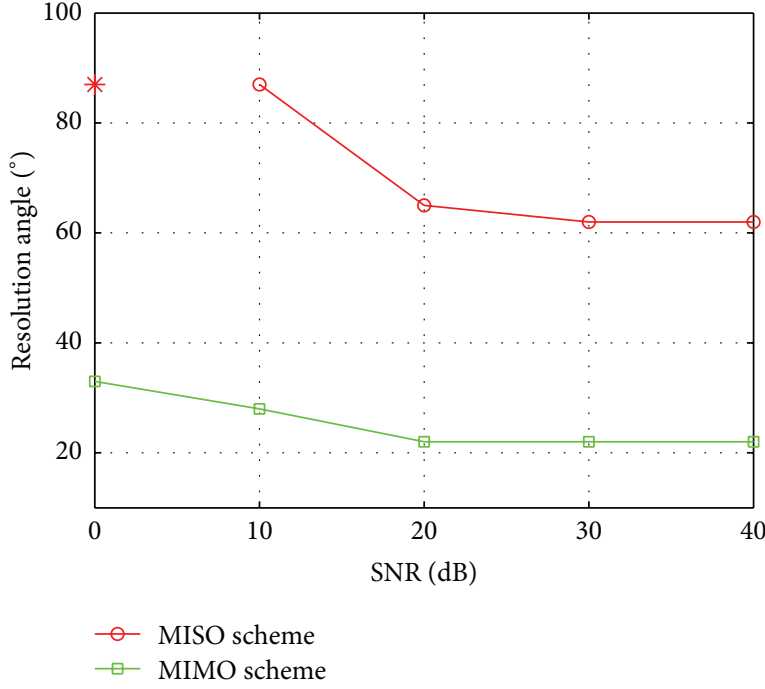

(a)

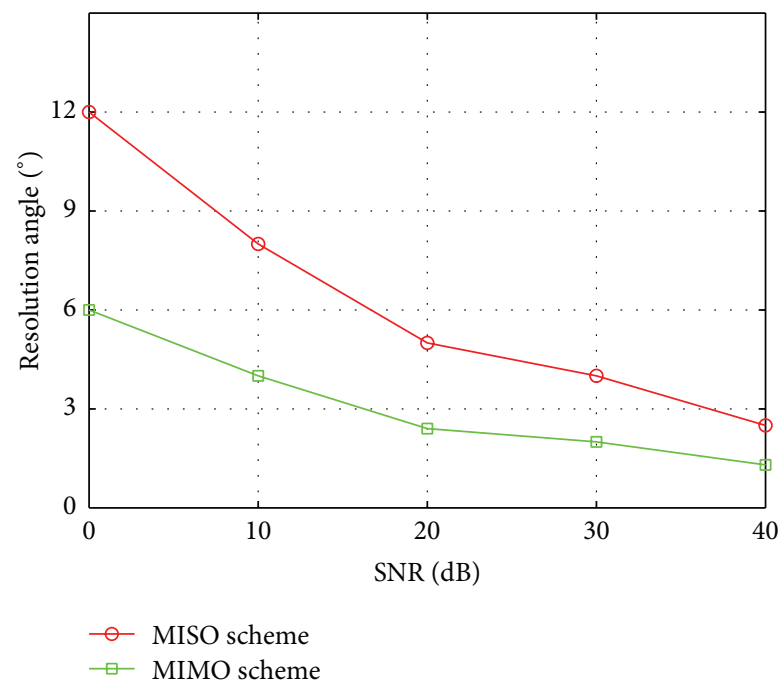

(b)

FIGURE 7: Resolution angle against SNR using (a) FFT and (b) MUSIC (* in (a) denotes no resolution ability for two targets). 


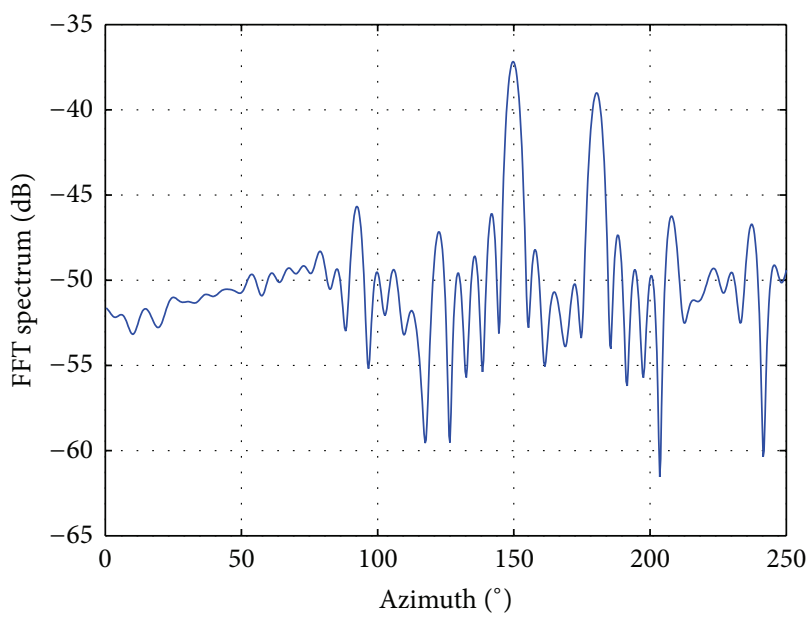

(a)

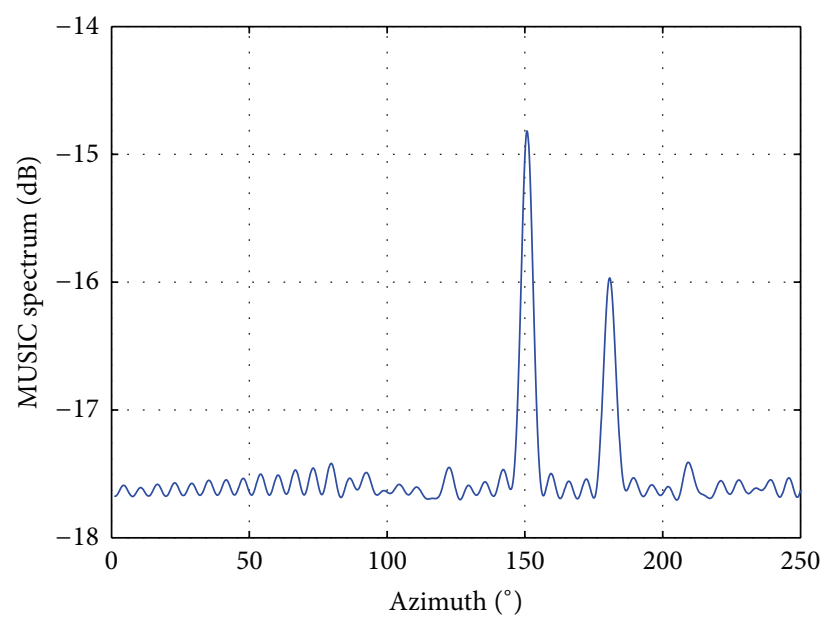

(b)

FIGURE 8: Azimuthal estimation for targets out of main lobes using (a) FFT and (b) MUSIC. Directions of two targets are $\left(34^{\circ}, 150^{\circ}\right)$ and $\left(34^{\circ}, 180^{\circ}\right)$.

robustness for detecting the targets out of the main lobes. Benefiting from the high-resolution ability, the same performance as the FFT was achieved by lower number of the OAM modes using the MUSIC algorithm and thus reduced the design complexity and cost of the hardware. The proposed model was built with the ideal propagation loss; however the gain loss for each OAM mode of the real system could be complex and would introduce the deviation. Further work could be focused on the calibration technique with measured propagation loss difference of each OAM mode to further reduce the estimation error.

\section{Competing Interests}

The authors declare that they have no competing interests.

\section{References}

[1] J. P. Torres and L. Torner, Twisted Photons: Applications of Light with Orbital Angular Momentum, John Wiley and Sons, 2011.

[2] A. M. Yao and M. J. Padgett, "Orbital angular momentum: origins, behavior and applications," Advances in Optics and Photonics, vol. 3, no. 2, pp. 161-204, 2011.

[3] B. Thidé, H. Then, J. Sjöholm et al., "Utilization of photon orbital angular momentum in the low-frequency radio domain," Physical Review Letters, vol. 99, no. 8, Article ID 087701, 2007.

[4] M. Barbuto, F. Trotta, F. Bilotti, and A. Toscano, "Circular polarized patch antenna generating orbital angular momentum," Progress in Electromagnetics Research, vol. 148, pp. 23-30, 2014.

[5] A. Bennis, R. Niemiec, C. Brousseau, K. Mahdjoubi, and O. Emile, "Flat plate for OAM generation in the millimeter band," in Proceedings of the 7th European Conference on Antennas and Propagation (EuCAP '13), pp. 3203-3207, Gothenburg, Sweden, April 2013.

[6] L. Cheng, W. Hong, and Z.-C. Hao, "Generation of electromagnetic waves with arbitrary orbital angular momentum modes," Scientific Reports, vol. 4, article 4814, 2014.
[7] D. K. Nguyen, O. Pascal, J. Sokoloff, A. Chabory, B. Palacin, and N. Capet, "Antenna gain and link budget for waves carrying orbital angular momentum," Radio Science, vol. 50, no. 11, pp. 1165-1175, 2015.

[8] S. M. Mohammadi, L. K. S. Daldorff, J. E. S. Bergman et al., "Orbital angular momentum in radioa system study," IEEE Transactions on Antennas and Propagation, vol. 58, no. 2, pp. 565-572, 2010.

[9] A. Tennant and B. Allen, "Generation of OAM radio waves using circular time-switched array antenna," Electronics Letters, vol. 48, no. 21, pp. 1365-1366, 2012.

[10] O. Edfors and A. J. Johansson, "Is orbital angular momentum (OAM) based radio communication an unexploited area?" IEEE Transactions on Antennas and Propagation, vol. 60, no. 2, pp. 1126-1131, 2012.

[11] F. Tamburini, E. Mari, A. Sponselli, B. Thidé, A. Bianchini, and F. Romanato, "Encoding many channels on the same frequency through radio vorticity: first experimental test," New Journal of Physics, vol. 14, no. 3, Article ID 033001, 2012.

[12] X. Hui, S. Zheng, Y. Chen et al., "Multiplexed millimeter wave communication with dual orbital angular momentum (OAM) mode antennas," Scientific Reports, vol. 5, article 10148, 2015.

[13] Y. Yan, G. Xie, M. P. J. Lavery et al., "High-capacity millimetrewave communications with orbital angular momentum multiplexing," Nature Communications, vol. 5, article 4876, 2015.

[14] G. Guo, W. Hu, and X. Du, "Electromagnetic vortex based radar target imaging," Journal of National University of Defense Technology, vol. 35, no. 6, pp. 71-76, 2013.

[15] K. Liu, Y. Cheng, Z. Yang, H. Wang, Y. Qin, and X. Li, "Orbitalangular-momentum-based electromagnetic vortex imaging," IEEE Antennas and Wireless Propagation Letters, vol. 14, pp. 711714, 2015. 
[16] T. Yuan, H. Wang, Y. Qin, and Y. Cheng, "Electromagnetic vortex imaging using uniform concentric circular arrays," IEEE Antennas and Wireless Propagation Letters, vol. 15, pp. 10241027, 2016.

[17] R. O. Schmidt, "Multiple emitter location and signal parameter estimation," IEEE Transactions on Antennas and Propagation, vol. 34, no. 3, pp. 276-280, 1986.

[18] S. U. Pillai and B. H. Kwon, "Forward/backward spatial smoothing techniques for coherent signal identification," IEEE Transactions on Acoustics, Speech, and Signal Processing, vol. 37, no. 1, pp. 8-15, 1989. 


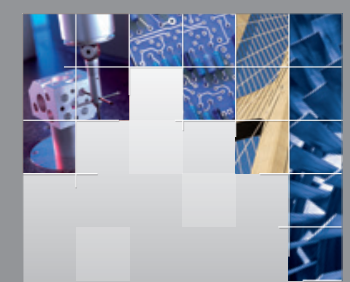

\section{Enfincering}
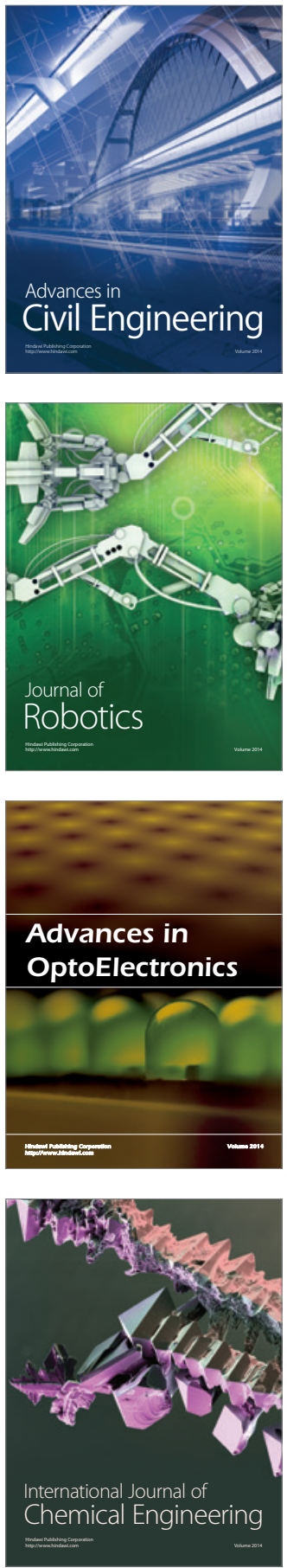

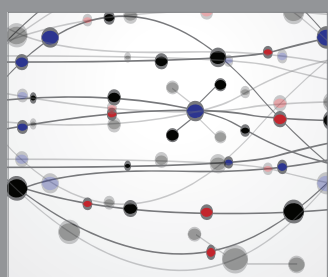

The Scientific World Journal

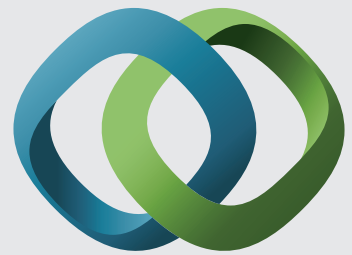

\section{Hindawi}

Submit your manuscripts at

http://www.hindawi.com
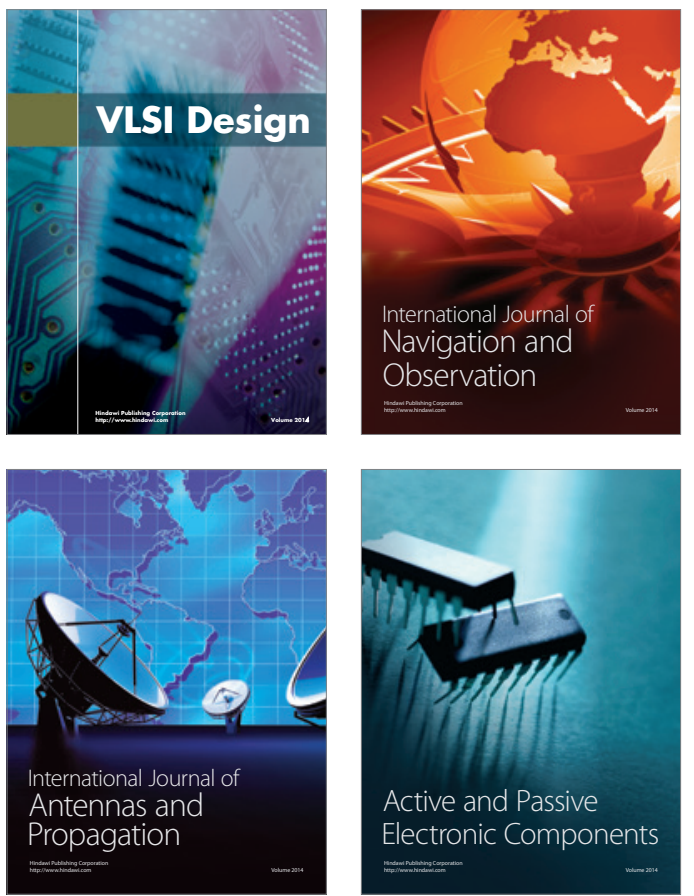
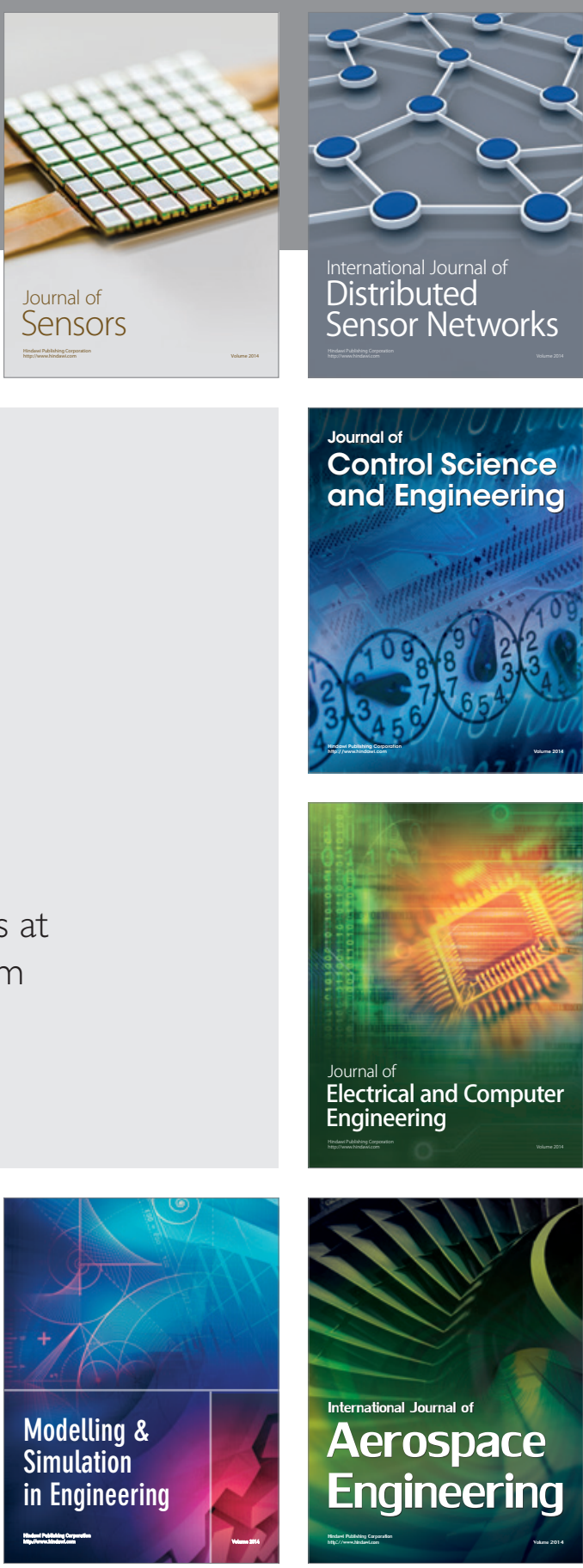

International Journal of

Distributed

Sensor Networks

Journal of

Control Science

and Engineering
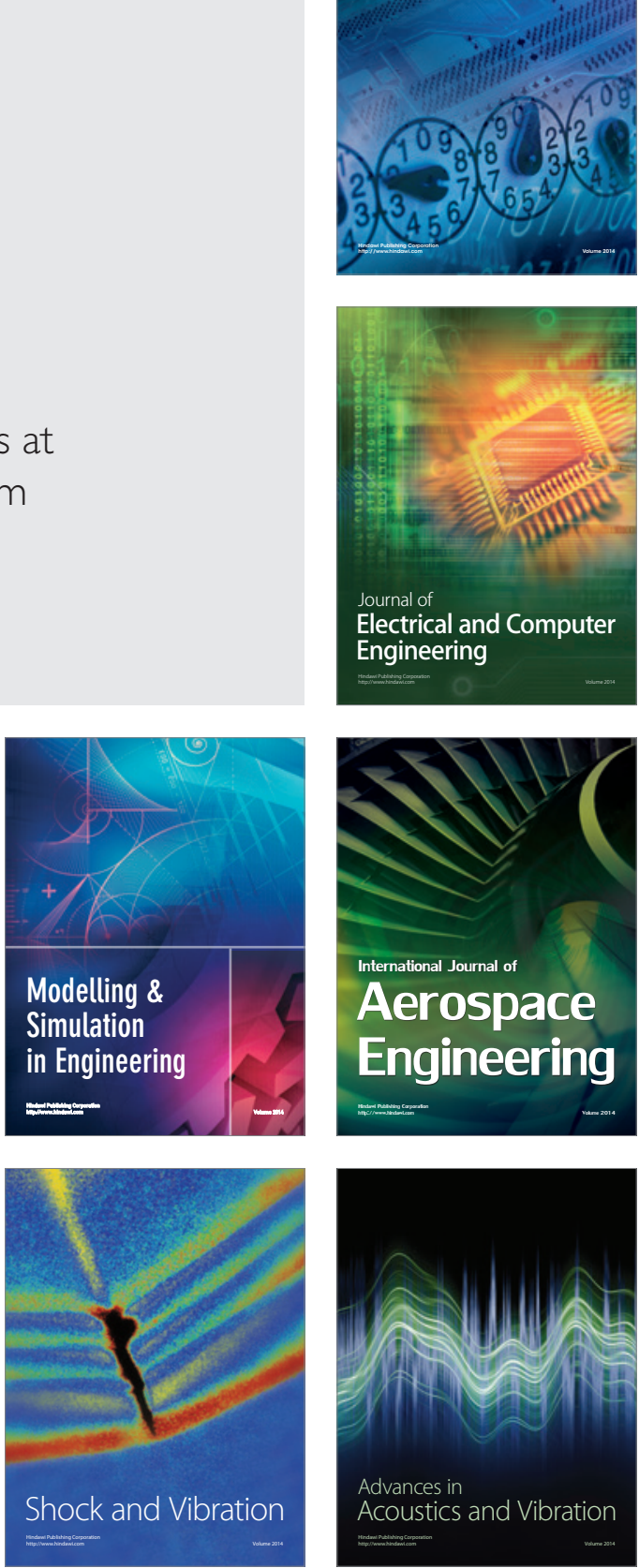